Results: We implemented the program at nine different sites in Mie prefecture, and a total of 40 medical and health professionals participated in the program. The program was well perceived and the participants expressed their willingness to undergo the exercise with other various scenarios on a regular basis.

Conclusion: A practical program with a useful framework to prepare health and medical coordinators in disaster prone areas was successfully developed and implemented. We believe the approach used in this program could help in training health professionals in disaster prone areas.

Prehosp Disaster Med 2017;32(Suppl. 1):s24-s25

doi:10.1017/S1049023X1700084X

Creating Order Out of Chaos: Centralized Team Training for Disaster and Austere Medical Response Richard P. Koebler ${ }^{1}$, Sierra Bourne ${ }^{2}$, Scott Mcguire ${ }^{3}$, Michael M. Karch ${ }^{4}$

1. Surgery, Mammoth Hospital, Mammoth Lakes/United States of America

2. Emergency Medicine, Mammoth Hospital, Mammoth Lakes/ $\mathrm{CA} /$ United States of America

3. Mammoth Medical Missions, Mammoth Lakes/CA/United States of America

4. Surgery, Mammoth Hospital, Mammoth Lakes/CA/United States of America

Study/Objective: To explore a prototype in the medical training for civilian disaster response.

Background: Medical response to complex humanitarian disasters requires organized training that is lacking in most civilian health care providers. We believe that because of the unique challenges faced in austere medical environments, a centralized team approach to training needs to be created by the overarching command structure of international disaster response. This training should include not only damage control procedural skill acquisition, as well as realistic simulation drills, but also fundamental instruction of the pre-existing command framework within the greater disaster response, such that a trained team can productively incorporate within this context.

Methods: Modeled after the military Tactical Combat Casualty Care (TCCC), we developed a team-based, Disaster and Austere Medicine Course for civilian providers called the International Disaster Austere Medicine Course (IDAMC). This course has been in existence for five years and highlights didactic teaching, procedural skills, simulation training, and Mass Casualty theory through the use of cadaver models and surgical simulators.

Results: Participants demonstrated an increased knowledge of core curricula learning objectives on pre- and post-course testing and displayed increased knowledge of their role within the structure of a greater disaster response. One disaster response team, in which $76 \%$ percent had undergone IDAMC training, was able to work efficiently in the immediate aftermath of Super Typhoon Haiyan and serve as the de facto hospital for a population of 2.1 million for four days.

Conclusion: The IDAMC serves as a prototype for civilian medical training in which simulation, procedural skills, and disaster response command framework are taught based on a successful military model.

Prehosp Disaster Med 2017;32(Suppl. 1):s25 doi:10.1017/S1049023X17000851

\section{Disaster Preparedness for Clinics - Further Study} from Haiti

Benjamin J. Kaufman ${ }^{1}$, Christina Bloem ${ }^{1}$, Sadia Hussain ${ }^{2}$, Matthew Riscint ${ }^{1}$, Bonnie Arquilla ${ }^{1}$

1. Emergency Medicine, SUNY Downstate, Brooklyn/United States of America

2. Massachusetts General Hospital, Boston/MA/United States of America

Study/Objective: Our team created a manual to train clinics in Low- and Middle-income (LMI) countries to effectively respond to disasters. This study is follow-up to a prior study evaluating disaster response. We returned to previously trained clinics to evaluate retention and performance in a disaster simulation.

Background: Local clinics are the first stop for patients when disaster strikes in LMI countries. They are often underresourced and under-prepared to respond to patient needs. Further effort is required to prepare these crucial institutions to respond effectively, using the Incident Command System (ICS) framework.

Methods: Two clinics in the North East Region of Haiti were trained through a disaster manual created to help clinics in LMI countries respond effectively to disasters. This study measured the clinic staffs response to a disaster drill using the Incident Command System (ICS) and compared the results to prior responses.

Results: Using the prior study's evaluation scale, clinics were evaluated on their ability to set up an Incident Command System. During the mock disaster, staff was evaluated on a 3 -point scale in 13 different metrics grading their ability to mitigate, prepare, respond and recover in a disaster. By this scale, both clinics were effective $(36 / 39,92 \%)$ in responding to a disaster.

Conclusion: The clinics retained much of the prior training and after repeated training the clinics improved their disaster response. Future study will evaluate the clinic's ability to integrate disaster response with regional health resources, to enable an effective outcome for patients.

Prehosp Disaster Med 2017;32(Suppl. 1):s25

doi:10.1017/S1049023X17000863

\section{Simulating a Disaster - Preparing Responders}

in India

Benjamin J. Kaufman ${ }^{1}$, Pia Daniel ${ }^{2}$, Bonnie Arquilla ${ }^{1}$, Joseph Freedman ${ }^{1}$, Bryan Jarrett ${ }^{1}$

1. Emergency Medicine, SUNY Downstate, Brooklyn/United States of America

2. Emergency Department, Columbia University Medical Center, New york city/NY/United States of America

Study/Objective: This study evaluates the effectiveness of a novel modality created by our team to teach disaster 